\title{
Characterization of rattling in relation to thermal conductivity: ordered half-Heusler semiconductors
}

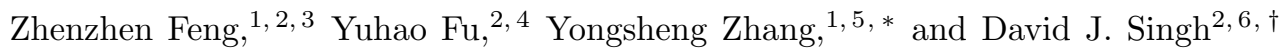 \\ ${ }^{1}$ Key Laboratory of Materials Physics, Institute of Solid State Physics, \\ Chinese Academy of Sciences, Hefei 230031, China \\ ${ }^{2}$ Department of Physics and Astronomy, University of Missouri, Columbia, MO 65211-7010, USA \\ ${ }^{3}$ Institute for Computational Materials Science, School of Physics and Electronics, Henan University, Kaifeng, 475004, China \\ ${ }^{4}$ College of Physics, Jilin University, Changchun 130012, China \\ ${ }^{5}$ Science Island Branch of Graduate School, University of Science and Technology of China, Hefei 230026, China \\ ${ }^{6}$ Department of Chemistry, University of Missouri, Columbia, MO 65211, USA
}

(Dated: January 23, 2020)

\begin{abstract}
The factors that affect the thermal conductivity of semiconductors is a topic of great scientific interest, especially in relation to thermoelectrics. Key developments have been the concept of the phonon-glass-electron-crystal (PGEC) and the related idea of rattling to achieve this. We use first principles phonon and thermal conductivity calculations in order to explore the concept of rattling for stoichiometric ordered half-Heusler compounds. These compounds can be regarded as filled zinc blende materials, and the filling atom could be viewed as a rattler if it is weakly bound. We use two simple metrics, one related to the frequency and the other to bond frustration and anharmonicity. We find that both measures correlate with thermal conductivity. This suggests that both may be useful in screening materials for low thermal conductivity.
\end{abstract}

\section{INTRODUCTION}

Thermal conductivity is an important primary quantity in describing the behavior of a material. It is of particular importance for thermoelectrics (TE), where low thermal conductivity, especially low lattice thermal conductivity is desired. The conversion efficiency is characterized by the thermoelectric figure of merit $Z T=\sigma S^{2} T /\left(\kappa_{e}+\kappa_{l}\right)$, where $S$ is the Seebeck coefficient, $\sigma$ is the electrical conductivity, $\kappa_{e}$ is the electronic thermal conductivity, $\kappa_{l}$ is the lattice thermal conductivity, and $T$ is the absolute temperature! ${ }^{1 / 2}$ The search for high $Z T$ led to the concept of the phonon-glass-electron crystal (PGEC), which is the idea of looking for semiconductors that have low electron scattering, and therefore high electrical conductivity, but at the same time very strong phonon scattering. 3 This together with the idea of rattling has been an influential and successful theme in TE research. It has led to the identification of many interesting novel materials, including clathrates and filled skutterudites! $3 \mid 5$

The purpose of this paper is to examine this concept in ordered stoichiometric half-Heusler compounds. The half-Heusler structure can be viewed as a filled zinc blende lattice. Therefore, if one of the atoms is weakly bound, one could imagine that it may serve as a rattler lowering the thermal conductivity. However, as discussed below, application of this concept to half-Heuslers is nontrivial, since they have rather complex lattice dynamics. Nonetheless, we do find that some compounds can be described as having rattling behavior in relation to thermal conductivity, and we discuss its characterization using two different measures.

The basic idea of rattling is to start with a semiconductor framework and fill with guest atoms that might strongly scatter heat carrying phonons of the host lattice, while maintaining the electronic structure and electronic transport. Realizations have invariably involved guest atoms that are bound in the host by chemical interactions, for example, bonding of the fillers in skutterudites, 1011 leading to modifications of the electronic structure. However, with careful selection these electronic changes can be beneficial for thermoelectric performance beyond the reduced $\kappa_{l}$ [12 14

This raises the questions of mechanism, how to identify rattling in a material, and how effective a given rattler may be in reducing thermal conductivity. Lattice thermal conductivity in normal crystalline materials is governed by a dispersion relation, i.e. the phonons, and scattering, which can have different contributions. For clean materials anharmonic three phonon umklapp scattering is often dominant in controlling thermal conductivity. 15

Guest atoms serving as rattlers may introduce low frequency vibrations that hybridize with the heat carrying acoustic modes. This will reduce the group velocity in the frequency range near the crossings of the acoustic branches with the guest atom vibrations due to hybridization with the rattler optic phonons. The resulting reduction in thermal conductivity is then a harmonic effect, arising from changes in dispersion due to hybridization of the vibrations of the host lattice and the guest atoms. In this view, the frequency of the rattling vibrations and the harmonic interaction with the host lattice plays the central role. This view has led to the development of some of the high performance skutterudites, where multiple fillers scatter acoustic phonons in different frequency ranges. 9

Another view is that strong anharmonicity associated with weak bonding is crucial. The strong anharmonicity then leads strong phonon scattering. Rattling systems often have weakly bonded atoms with long, stretched bonds and large atomic displacement parameters (large 
mean square displacements), which have been used to characterize this type of behavior $\frac{16}{}$

These two views (harmonic interactions mixing modes vs. strong anharmonicity due from weak bonding) are seemingly divergent, and have been the source of some controversy, e.g. in skutterudites $\frac{17 \mid 18}{18}$ They are in fact different. However, they are complementary in that both mechanisms may be operative in a given system, and both may be ways of achieving low thermal conductivity.

Importantly, recently developed thermal conductivity methods based on anharmonic phonon scattering allow one to directly calculate lattice thermal conductivity ${ }^{[15] 19}[22]$ Here we use these tools in conjunction with simple parameters that can be extracted from phonon dispersions to explore these different views and to find phonon based metrics that may be useful in identifying rattling and low thermal conductivity without direct thermal conductivity calculations.

As mentioned, we use half-Heusler semiconductors for this purpose. This is a very large class of compounds that has a simple crystal structure and contains many known good thermoelectric materials. $\frac{3223}{30}$ This family shows considerable chemical flexibility, as reflected in the large number of compounds. Half-Heusler compounds also exhibit a very large range of thermal conductivities ${ }^{31}$ The structure can be regarded as a filled zinc blende structure, which suggests possibilities for rattling if the filling atom is weakly bound. The interest in half-Heusler TE materials has motivated much work on and current interest in their thermal conductivities and ways of minimizing them ${ }^{31}+39$ Furthermore, as recently discussed by Berland and co-workers, $\frac{39}{39}$ their thermal properties are very subtle. Besides the large range of thermal conductivities that can occur, the low thermal conductivity compounds of interest for thermoelectrics, can have sizable reductions in lattice thermal conductivity due to disorder and grain boundaries, as well as substantial electronic contributions, ${ }^{[39}$ which are, however, highly non-trivial to extract from experimental data alone. $\frac{40}{}$ In addition, there are several mechanisms that can be important for reducing the thermal conductivity of half-Heusler compounds. These include site disorder, alloy scattering, and anharmonicity related to lone pair physics (also discussed as resonant bonding), 41 and other features of the bonding that lead to anharmonicity. ${ }^{[39 \mid 42}$ Here we examine the concept of rattling in order to characterize it in the context of these materials and to examine the extent to which and how this concept can be applied.

\section{STRUCTURE AND METHODS}

Half-Heuslers are ternary intermetallics with general formula $\mathrm{ABC}$, occurring in cubic space group F $\overline{4} 3 \mathrm{~m}$ (Figure 11). The structure consists of three interpenetrating face centered cubic (fcc) sublattices and one vacant fcc sublattice, which if filled would yield the full-Heusler structure. Here we follow the common notation, where

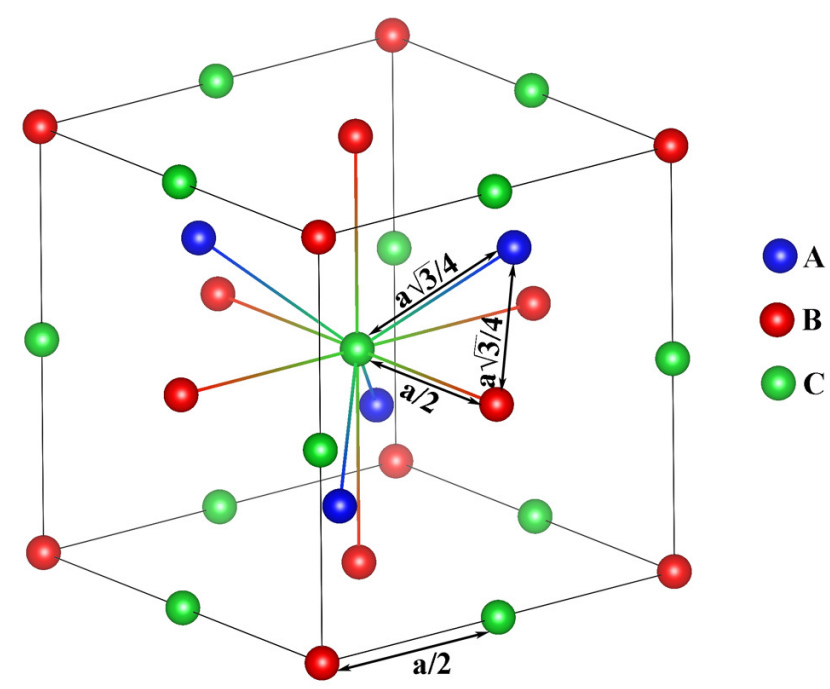

FIG. 1. The half-Heusler crystal structure, showing the bond lengths. Note in particular the two bond identical bond lengths of $\sqrt{3} a / 4$ even though the atoms involved are different.

the A occupies 4c $(1 / 4,1 / 4,1 / 4)$, B occupies $4 \mathrm{a}(0,0$, $0)$ and $\mathrm{C}$ occupies $4 \mathrm{~b}(1 / 2,1 / 2,1 / 2)$, rather than IU$\mathrm{PAC}$ notation in order to clearly connect with the structures. In terms of coordination, each A atom has four B neighbors and four $\mathrm{C}$ neighbors at distance $a \sqrt{3} / 4$. The $\mathrm{B}$ and $\mathrm{C}$ atoms are each coordinated by four A neighbors at a distance of $a \sqrt{3} / 4$. We note that exchanging the $\mathrm{A}$ atom with $\mathrm{B}$ or $\mathrm{C}$ results in a different material. Here, we checked each possibility using the total energy and performed calculations for the lowest energy ordering. The results are consistent with the report of Carrete and coworkers. 31

The half-Heusler structure has a single structural parameter, the lattice parameter $a$, but two distinct short nearest neighbor bonds. This plus the wide chemical flexibility of the structure type provides opportunities for having compounds with frustrated bond lengths, and therefore potentially rattling atom physics. However, simple measures of bond satisfaction, e.g. comparisons of sums of ionic radii with bond lengths, as have been highly successful in understanding the structures of oxides, are difficult to apply in half-Heusler compounds because of the variety of different bonding types (metallic, covalent, ionic and mixtures) that occur in this family $43-45$ Goals of the present work include finding ways of describing rattling based on the idea of bond length frustration and examining the extent to which these measures, and therefore the idea of rattling due to bond frustration underlies the exceptional range of thermal conductivities found in half-Heusler semiconductors.

For this purpose we use a set of 75 known and potential half-Heusler compounds that were identified by Carrete and co-workers, ${ }^{31}$ and were previously used in other screens related to thermoelectrics, including thermal and 
electronic properties, and searches for other systems. $\stackrel{46 / 47}{4}$ This list includes both known and hypothetical materials predicted by stability analysis.

We calculated the phonon dispersions for all 75 of the proposed half-Heusler compounds from the data set. We find that 74 of them have phonon dispersions with only stable modes, and use these compounds as the set to analyze. SbNaSr is found to be dynamically unstable. We then directly calculated the lattice thermal conductivity of each compound by solving the linearized BoltzmannPeierls transport equation with the ShengBTE package. This gives us a set of phonon calculations, which are the basis for our analysis, and a set of thermal conductivities. For some compounds that had low thermal conductivities, we did additional calculations using the temperature dependent effective potential method to generate the anharmonic coefficients for the Boltzmann transport calculations. This provides a more stable and presumably more accurate result for such cases, but uses the same underlying three-phonon scattering physics for the thermal conductivity.

Our density functional calculations were done using the projector augmented wave (PAW) method, ${ }^{48}$ as implemented in the VASP code ${ }^{49}$ We used the generalized gradient approximation of Perdew, Burke and Ernzerhof (PBE-GGA), with an energy cutoff of $500 \mathrm{eV}$ and Brillouin zone samples based on a $10 \times 10 \times 10$ mesh.

The thermal conductivity calculations were done with standard iterative solution of the Boltzmann equation $\underline{50}$ with harmonic and anharmonic interatomic force constants from density functional theory. All contributions from two-phonon and three-phonon scattering processes were included. For the cubic systems considered here, $\kappa_{l}$ is a scalar quantity given by

$$
\kappa_{l} \equiv \kappa_{l}^{\alpha \alpha}=\frac{1}{N V} \sum_{\lambda} C_{\lambda} v_{\lambda}^{\alpha} v_{\lambda}^{\alpha} \tau_{\lambda}
$$

Where $\lambda$ denotes a phonon mode in branch $p$ with wave vector $\boldsymbol{q}, N$ is the number of uniformly spaced $\boldsymbol{q}$ points in the phonon BZ, $V$ is the volume of the unit cell, $C_{\lambda}$ is the specific heat, $v_{\lambda}$ is the phonon group velocity, and $\tau_{\lambda}$ is the lifetime with an applied temperature gradient along the $\alpha$ direction. $\tau_{\lambda}$ is determined by the processes of two-phonon scattering from isotopic disorder and threephonon anharmonic scattering. Here we focus on threephonon anharmonic scattering. $\tau_{\lambda}$ is given by the sum of all possible transition probabilities for mode $\lambda$ with modes $\lambda^{\prime}$ and $\lambda^{\prime \prime}$,

$$
\begin{aligned}
\Gamma_{\lambda \lambda^{\prime} \lambda^{\prime \prime}}^{ \pm}= & \frac{\hbar}{8 N_{0}}\left\{\begin{array}{c}
n_{\lambda^{\prime}}^{0}-n_{\lambda^{\prime \prime}}^{0} \\
n_{\lambda^{\prime}}^{0}+n_{\lambda^{\prime \prime}}^{0}+1
\end{array}\right\} \\
& \times\left|\Phi_{\lambda \lambda^{\prime} \lambda^{\prime \prime}}\right|^{2} \frac{\delta\left(\omega_{\lambda} \pm \omega_{\lambda^{\prime}}-\omega_{\lambda^{\prime \prime}}\right)}{\omega_{\lambda} \omega_{\lambda^{\prime}} \omega_{\lambda^{\prime \prime}}}
\end{aligned}
$$

that satisfy momentum and energy conversation. where the upper (lower) row is curly brackets go with the $+(-)$ signs are for absorption (emission) processes. $\omega_{\lambda}$ is the angular frequency corresponding to the $\lambda$ th mode, and $\left|\Phi_{\lambda \lambda^{\prime} \lambda^{\prime \prime}}\right|^{2}$ are the scattering matrix elements,

$$
\begin{aligned}
\Phi_{\lambda \lambda^{\prime} \lambda^{\prime \prime}}= & \sum_{k} \sum_{l^{\prime} k^{\prime}} \sum_{l^{\prime \prime} k^{\prime \prime}} \sum_{\alpha \beta \gamma} \Phi_{\alpha \beta \gamma}\left(0 k, l^{\prime} k^{\prime}, l^{\prime \prime} k^{\prime \prime}\right) \\
& \times \frac{e_{\alpha k}^{\lambda} e_{\beta k^{\prime}}^{\lambda^{\prime}} e_{\gamma k^{\prime \prime}}^{\prime \prime}}{\sqrt{M_{k} M_{k^{\prime}} M_{k^{\prime \prime}}}} e^{i q^{\prime} R_{l^{\prime}}} e^{i q^{\prime \prime} R_{l^{\prime \prime}}}
\end{aligned}
$$

where $M_{k}$ is the atomic mass of the $k$ th atom, and $\Phi_{\alpha \beta \gamma}\left(0 k, l^{\prime} k^{\prime}, l^{\prime \prime} k^{\prime \prime}\right)$ are the anharmonic interatomic force constants (IFCs). Then the phonon angular frequencies $\omega_{\lambda}$ are obtained from diagonalization of the dynamical matrix.

The phonon dispersions and the harmonic secondorder interatomic force constants (IFCs) were calculated using the frozen phonon method, as implemented in the Phonopy package 51 4 $4 \times 4 \times 4$ supercells (with 192 atoms in total) and $2 \times 2 \times 2$ supercell $k$-meshes were used for the dynamic matrix. As mentioned, $\mathrm{SbNaSr}$ is found to be dynamically unstable, and is not further considered here. The anharmonic IFCs were calculated using the same supercell and $k$-mesh. The ShengBTE package $\frac{19120}{}$ was employed to iteratively solve the phonon Boltzmann equation. $15 \times 15 \times 15$ q-grids were used. In additional for low thermal conductivity compounds, which are of particular interest for this study, we did ab initio molecular dynamics (AIMD). This allows determination of temperature dependent harmonic and anharmonic interatomic force constants.

This can be different from static calculations for highly anharmonic materials, i.e. materials that have low thermal conductivity due to strong anharmonicity. We calculated thermal conductivities for PtLaSb, SiAlLi, BiBaK, PCdNa, CoAsHf, PdBiSc, GeAlLi, and ZnLiSb using this temperature-dependent effective potential (TDEP) method $!^{52}$ For this purpose we employed Born-Oppenheimer molecular dynamics with the PAW method, as implemented in the VASP code. The parameters were similar to the ground state calculations, except that a somewhat lower planewave cutoff of $330 \mathrm{eV}$ was used. The simulations were run for approximately 100 ps with a time step of $1 \mathrm{fs}$ and a temperature of $300 \mathrm{~K}$, which is the temperature that we focus on in this study.

The use of TDEP gives significant differences from ShengBTE results for only three compounds, specifically, SiAlLi, CoAsHf, and ZnLiSb. Therefore, in the following we report the data calculated by ShengBTE except for these three compounds (SiAlLi, CoAsHf, and ZnLiSb), for which TDEP results are used.

\section{RESULTS AND DISCUSSION}

The calculated thermal conductivities for the 74 halfHeusler compounds are listed in TableI. This is the basis for the comparisons given in the remainder of this paper. As seen in Table I four compounds have very low $300 \mathrm{~K}$ 
TABLE I. Calculated lattice thermal conductivities of 74 half-Heusler compounds at $300 \mathrm{~K}$, in units of W/mK

\begin{tabular}{|c|c|c|c|c|c|c|c|}
\hline Comp. & $\kappa_{l}$ & Comp. & $\kappa_{l}$ & Comp. & $\kappa_{l}$ & Comp. & $\kappa_{l}$ \\
\hline PtLaSb & 0.84 & PdHfSn & 14.76 & RuTeZr & 19.61 & CoGeNb & 26.99 \\
\hline RhLaTe & 1.21 & NiAsSc & 15.76 & CoNbSn & 20.06 & OsSbTa & 28.12 \\
\hline BiBaK & 2.26 & $\mathrm{NiPbZr}$ & 15.94 & CoSbTi & 20.34 & $\mathrm{IrGeV}$ & 28.35 \\
\hline $\mathrm{PCdNa}$ & 2.27 & RhAsZr & 16.22 & RuSbTa & 20.45 & IrGeNb & 28.71 \\
\hline $\mathrm{ZnLiSb}$ & 7.03 & CoBiZr & 16.70 & CoGeTa & 20.73 & CoSiTa & 28.87 \\
\hline CoAsTi & 7.28 & $\mathrm{CoSnV}$ & 16.83 & CoAsZr & 20.82 & OsNbSb & 29.38 \\
\hline $\mathrm{NiBiY}$ & 7.44 & RhSnTa & 16.99 & NiGeTi & 20.88 & $\mathrm{FeNbSb}$ & 29.61 \\
\hline IrAsZr & 7.70 & CoNbSi & 17.06 & PtGeTi & 21.61 & FeSbTa & 30.28 \\
\hline PdBiSc & 7.82 & PdGeZr & 17.13 & IrAsTi & 21.79 & IrGeTa & 32.19 \\
\hline $\mathrm{PdPbZr}$ & 9.42 & FeGeW & 17.16 & CoSnTa & 21.79 & AuAlHf & 33.85 \\
\hline CoHfSb & 10.01 & SiAlLi & 17.19 & NiGeZr & 22.28 & $\mathrm{RuAsNb}$ & 36.08 \\
\hline RhBiHf & 10.46 & RhNbSn & 17.57 & $\mathrm{NiGaNb}$ & 22.84 & $\mathrm{FeAsNb}$ & 37.58 \\
\hline CoSbZr & 10.96 & GeAlLi & 17.71 & FeSbV & 23.01 & BLiSi & 37.71 \\
\hline IrBiZr & 11.15 & CoAsHf & 17.95 & $\mathrm{CoGeV}$ & 24.30 & IrSnTa & 78.09 \\
\hline RhBiTi & 11.41 & NiGeHf & 18.08 & FeAsTa & 24.55 & & \\
\hline NiBiSc & 11.56 & NiHfSn & 18.28 & PtGaTa & 24.55 & & \\
\hline RhBiZr & 12.45 & IrNbSn & 18.62 & RuNbSb & 24.91 & & \\
\hline PtGeZr & 12.68 & CoBiHf & 18.74 & RhAsTi & 24.94 & & \\
\hline $\mathrm{NiSnZr}$ & 13.26 & IrHfSb & 18.96 & RuAsTa & 25.58 & & \\
\hline NiSnTi & 14.63 & FeTeTi & 19.47 & CoBiTi & 26.67 & & \\
\hline
\end{tabular}

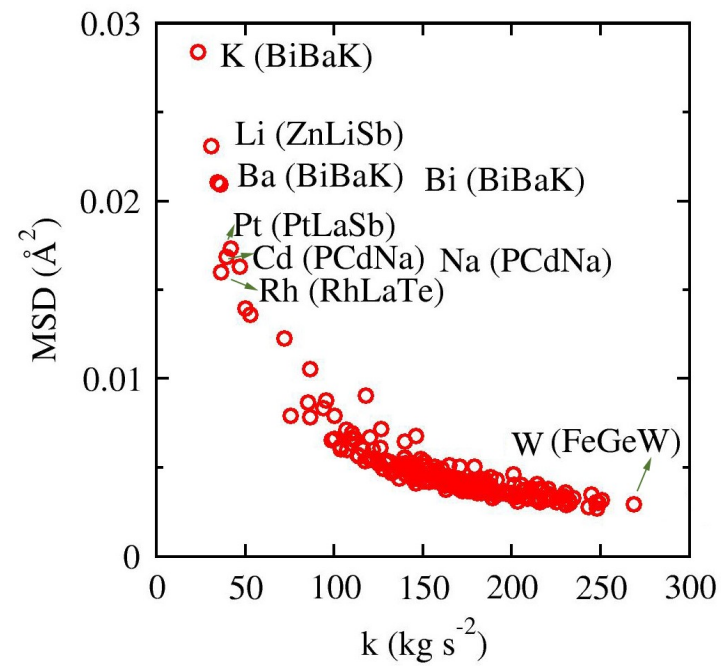

FIG. 2. Calculated mean square displacement (MSD) at 300 $\mathrm{K}$ vs. effective spring constant for the atoms in our dataset. Labels denote specific atoms in the compounds in parentheses, e.g. Li (ZnLiSb) denotes the Li in ZnLiSb.

lattice thermal conductivity, PtLaSb, RhLaTe, BiBaK, and PCdN, with $\kappa_{l}$ of $0.84 \mathrm{~W} / \mathrm{mK}, 1.21 \mathrm{~W} / \mathrm{mK}, 2.26$ $\mathrm{W} / \mathrm{mK}$, and $2.27 \mathrm{~W} / \mathrm{mK}$, respectively.

There are also many compounds with much higher thermal conductivity, ranging up to $78 \mathrm{~W} / \mathrm{mK}$ in the case of IrSnTa. The wide range of thermal conductivities implies that thermal conductivity of half-Heusler compounds involves rather rich physics related to phonon scattering. This wide range cannot be understood just by invoking measures based on the masses of the atoms involved or the acoustic phonon group velocities. For example, the lowest thermal conductivity material, PtLaSb, with $\kappa_{l}$ of $0.84 \mathrm{~W} / \mathrm{mK}$ has a mass per unit cell of $M_{\text {cell }}=455.75 \mathrm{amu}$, while IrSnTa has $M_{\text {cell }}=491.88 \mathrm{amu}$, i.e. close to, and even slightly larger than that of PbLaSb. Other measures are discussed below.

The half-Heusler structure contains three different atoms. If one of the atoms is very small, bound loosely and/or provides low optic phonon frequencies, it might be considered as a rattler. We constructed two measures based on the phonon dispersions. We note that phonon dispersions are much easier to calculate than thermal conductivities. In the following we explore ways of using these to understand thermal conductivity and identify materials with potentially low thermal conductivity. These measures are based on the atom projected phonon density of states. The first measure is from an average phonon frequency for each atom, calculated using the first frequency moment of the projected density of states. The idea is that if one atom has a much lower average frequency than the other two, or than the average frequency of the solid, then it might be a rattler. This measure connects with the idea that associates rattling with the introduction of low frequency optic branches than intersect with the heat carrying acoustic branches, 

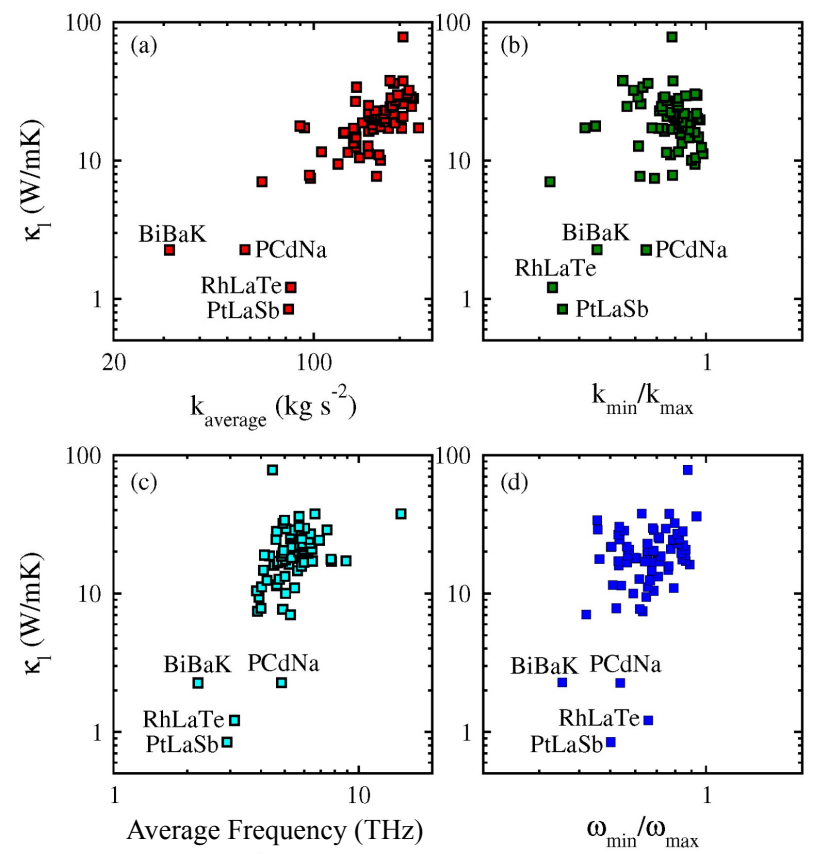

FIG. 3. (a) The average spring constant, $k_{\text {average }}$ as a function of lattice thermal conductivity for half-Heuslers. (b) The ratio of $k_{\min }$ to $k_{\max }$, here $k_{\min }$ and $k_{\max }$ are the smallest and largest spring constants among three atoms, respectively. (c) The average frequency, and corresponding the lattice thermal conductivity (here the average is calculated using the total DOS). (d) The ratio of $\omega_{\min }$ to $\omega_{\max }$, here $\omega_{\min }$ and $\omega_{\max }$ are the smallest and largest angular frequency among three atoms.

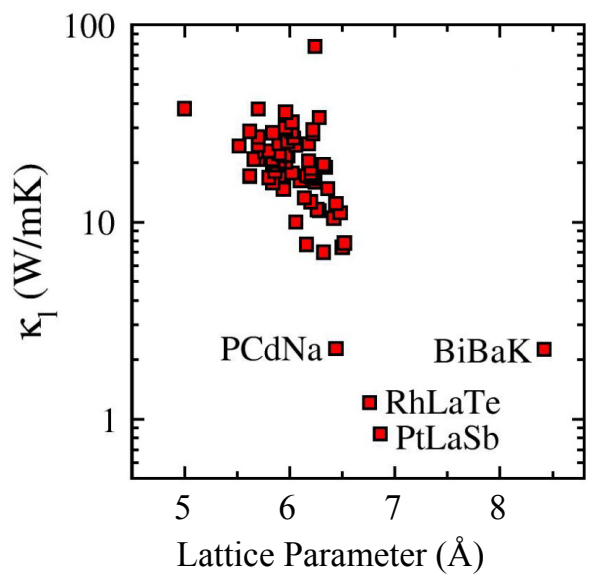

FIG. 4. Thermal conductivity and lattice parameter for half-Heusler compounds.

scattering acoustic phonons and modifying the harmonic acoustic phonon dispersions to reduce thermal conductivity. This point of view was emphasized in the context of skutterudites by Feldman and others 9918

A complementary point of view, discussed in the same context by Keppens and co-workers, $\frac{17}{17}$ is that strong anharmonicity is key. This is more difficult to character-
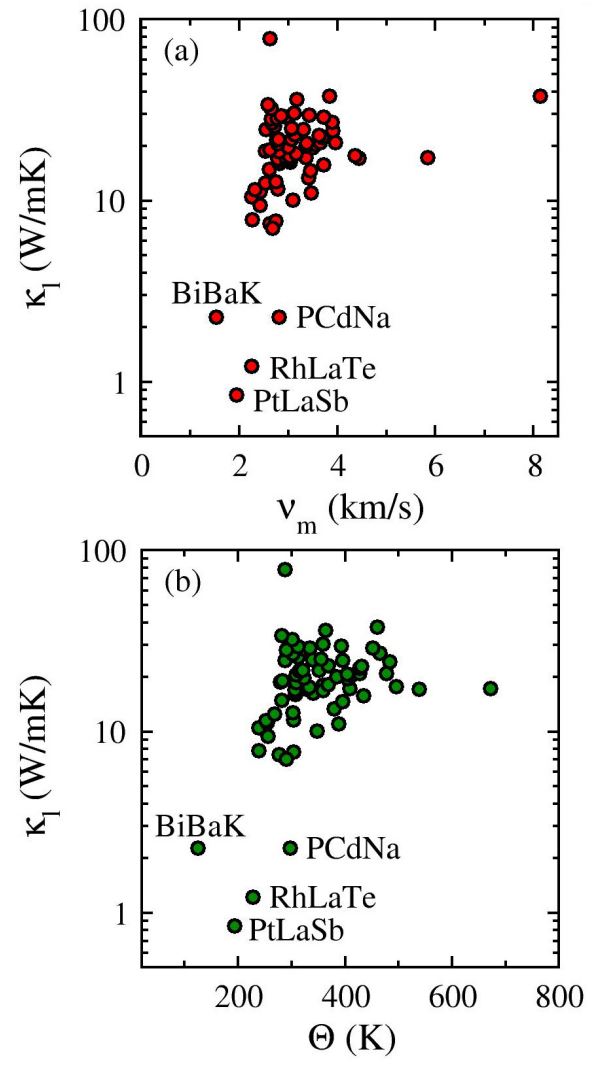

FIG. 5. Relationship of thermal conductivity with (a) the calculated average sound velocities and (b) the Debye temperature.

ize from the harmonic phonon frequencies. Here, we examine this using the concept where stretched bonds lead to strong anharmonicity, and characterize stretched bonds by low force constants. In particular, we compute an average spring constant for each atom, $\alpha$, using the average angular frequency, $\bar{\omega}_{\alpha}$, obtained as above, so that $k_{\alpha}=\bar{\omega}^{2} m_{\alpha}$, where $m_{\alpha}$ is the mass of the atom. We also define an average spring constant, which is just $k_{\text {average }}=\left(k_{1}+k_{2}+k_{3}\right) / 3$. This is different from the average that would be obtained from the average phonon frequency, due to the power of two in the formula for the spring constant.

As mentioned, large mean square displacements have been associated with rattling. All sites in the halfHeusler structures have cubic site symmetry. Therefore at the harmonic level the mean square displacements are isotropic. The mean square displacements (MSD) are expected to be closely related to the effective spring constant, $\mathrm{MSD} \propto 1 / k_{\alpha}$ due to equipartition at temperatures high enough that quantum effects are not important. This in fact is the case, as shown in Fig. 2, where the MSD for all atoms in the data set is plotted vs. effective spring constant from the phonon density of states.

Fig. 3(a) shows the thermal conductivity versus the $k_{\text {average. }}$ As may be expected, $k_{\text {average }}$ has a positive 
correlation with $\kappa_{l}$, which is simply a reflection of the fact that a stiff lattice favors high thermal conductivity. This is simply understood in terms of the Callaway model, $\stackrel{53}{[3}$ where thermal conductivity is proportional to a product of specific heat, phonon group velocity and phonon mean free path. A stiff lattice yields high phonon frequencies, and therefore high phonon group velocities. This leads to high $\kappa_{l}$ due to the proportionality of $\kappa_{l}$ and velocity.

A similar correlation is seen with the average frequency, though the separation of the high thermal conductivity compounds is actually somewhat weaker, even though it would seem at first glance that it should be better due to the more direct connection of frequency with the Callaway picture. The difference between these is in the mass, in other words the fact that lattices with heavy atoms have lower phonon frequencies and sound velocities, for the same force constants. This suggests that in fact force constants are important, perhaps because they also may contain chemical information about anharmonicity. As mentioned above, the mass itself does not explain the wide range of thermal conductivities. We also note that there is a correlation between thermal conductivity and the lattice parameter, as shown in Fig. 4. This reflects the idea that larger lattice parameter corresponds to weaker bonding in general. None of these correlations is strong enough to be used as a reliable predictor of thermal conductivity by itself.

The ratios of $k_{\min }$ to $k_{\max }$ are shown in Fig. 3(b). Here $k_{\min }$ and $k_{\max }$ are the smallest and largest effective spring constants among the three atoms. It is useful to remember that the half-Heusler structure can be regarded as a filled zinc blende structure. In the two atom zinc blende structure there is an expectation that the effective spring constants for the two atoms are generally similar. This is because they would necessarily be identical only nearest neighbor interactions were present. Small ratios of $k_{\min }$ to $k_{\max }$ mean that one atom is weakly bonded relative to the the others. Weak bonding of an atom will lower the sound velocity because the average stiffness of the lattice will be reduced, which can be expected to reduce the thermal conductivity. It will also lead to an atom whose motion becomes decoupled from the other atoms, leading to low frequency Einsteinlike phonon branches. This connects intuitively with the concept of rattling, which as mentioned can have strong effects on the thermal conductivity.

Crystallographic atomic displacement parameters (ADP, i.e. from experimental Debye-Waller factors), and calculated MSD have been discussed as an indicator of low thermal conductivity 16154 The ADP at a given temperature is determined by the effective spring constant, and not the frequency, since heavier mass will lower the frequency but will not increase the ADP.

We now discuss some other quantities that are commonly discussed in the context of thermal conductivity. The average frequency and the ratio of $\omega_{\min }$ to $\omega_{\max }$ are shown in Fig. 3(c) and (d). Not surprisingly there is a correlation between the average phonon frequency and $\kappa_{l}$. Importantly, there is also a clear correlation of $\kappa_{l}$ with the ratio. This relates to the idea of rattling in terms of an atom giving low frequency optic modes. Specifically, the ratio is not connected with the average sound velocity and therefore would naively not be a key parameter from the viewpoint of the Callaway expression.

The Debye temperature is another measure related to average phonon frequency. There are different definitions of the Debye temperature related to what quantity is being measured, e.g. specific heat, mean square displacements (x-ray Debye temperature) etc. Considering that thermal conductivity is often discussed in terms of acoustic modes, we calculated the elastic Debye temperature, which is the specific heat Debye temperature given by the elastic constants. This Debye temperature would not be affected by low lying optic modes such as those introduced by rattlers, except to the extent that weakly bound atom would reduce the overall elastic stiffness of the lattice. We obtained the the Debye temperature $\Theta$, and the average sound velocity $v_{m}$. The correlation of $\kappa_{l}$ with these quantities. is shown in Fig. 5. The Callaway expression applied to acoustic modes would suggest a strong correlation between average velocity and $\kappa_{l}$. Our results show that, while correlated, this not as strong a correlation as one might expect. In fact, it is similar to the correlation with $\Theta$, although the $\Theta$ also has a dependence on lattice parameter.

From our calculations, we find that PtLaSb, RhLaTe, $\mathrm{BiBaK}$, and $\mathrm{PCdNa}$ have very low lattice thermal conductivity. The phonon dispersions and the projected phonon densities of states of these four compounds are given in Fig. 6. The average phonon frequencies for each atom and the effective spring constants $k_{\alpha}$ for these four compounds are listed in Table III. The phonon dispersions of these four compounds show a strong difference between transverse and and longitudinal acoustic modes. In other words the transverse acoustic branches have much lower velocity than the longitudinal branches. While this is a commonly observed characteristic of materials, it is not a general characteristic of the zinc blende structure, from which the half-Heusler structure is derived. In the zinc blende semiconductors, the transverse branches are typically stiff reflecting bond bending forces from covalent bonding. This is known to lead to higher thermal conductivity, as in $\mathrm{BaAs}, \frac{55}{5}$ while conversely materials that have low velocity transverse branches, such as $\mathrm{CuCl}$, generally have low thermal conductivity 56

The phonon densities of states for the four compounds indicate that the low-energy acoustic modes are dominated by one atom $(\mathrm{Pt} / \mathrm{Rh} / \mathrm{Bi} / \mathrm{Cd})$ vibrations, whereas optical branches are governed by another two atoms. Moreover, there are avoided crossings of the longitudinal acoustic branch and optical phonon branches, which are a characteristic of rattling. The anharmonic scattering rates, which include both the effect of anharmonicity and the scattering phase space are clearly important for thermal conductivity, These are shown in Fig. 7 for the four compounds. The scattering rates for low frequency 

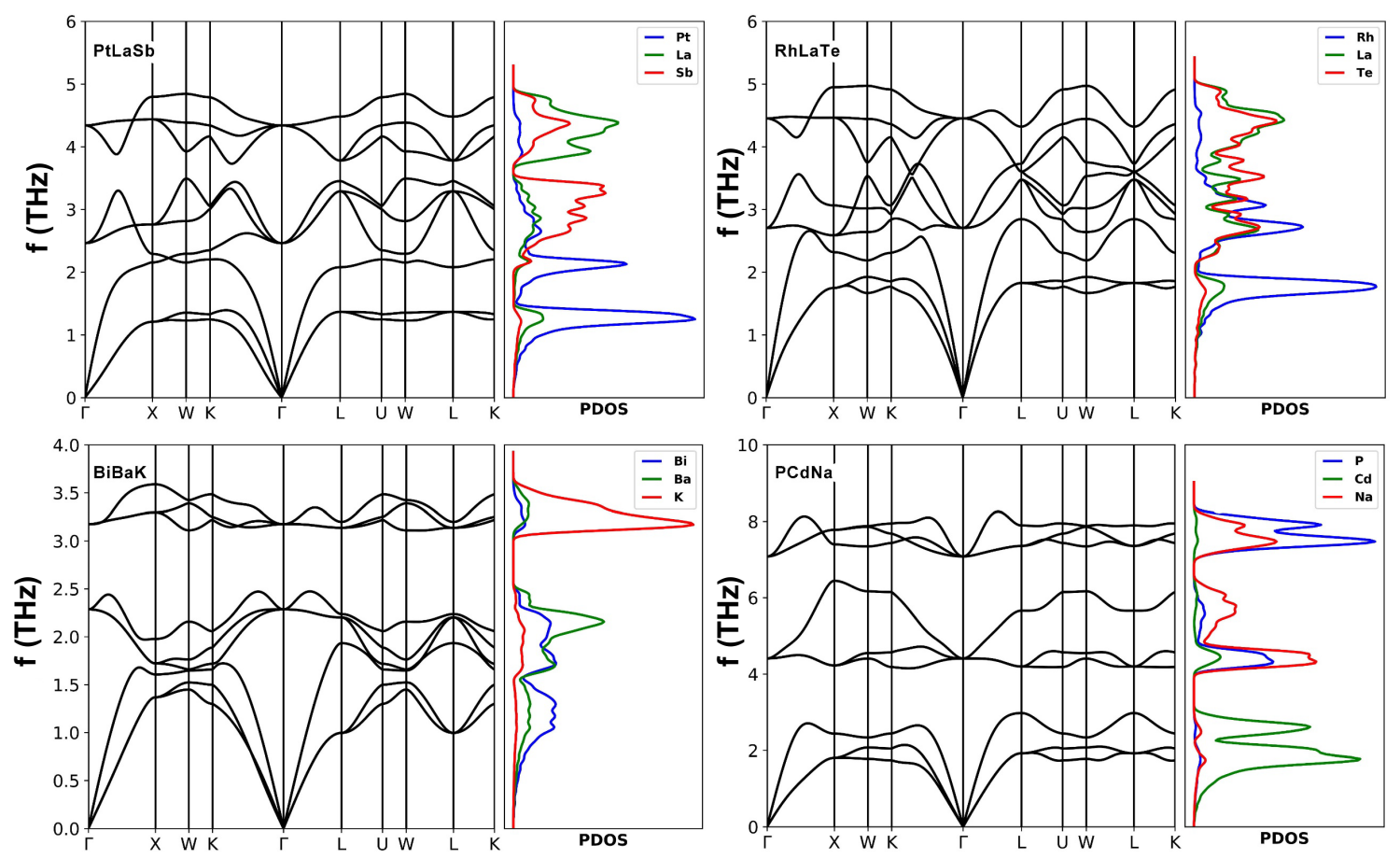

FIG. 6. Phonon dispersions and projected phonon density of states for PtLaSb, RhLaTe, BiBaK and PCdNa. Note the different frequency scales for different compounds.

TABLE II. Phonon frequency $f(\mathrm{THz})$, the spring constant $k(\mathrm{~N} / \mathrm{m})$ for each atom, the ratio between the minimum to maximum phonon frequency and spring constant in PtLaSb, RhLaTe, BiBaK, PCdNa.

\begin{tabular}{cccccccccccc}
\hline \hline Comp. & $f_{1}$ & $f_{2}$ & $f_{3}$ & $f_{\text {average }}$ & $f_{\min } / f_{\max }$ & $k_{1}$ & $k_{2}$ & $k_{3}$ & $k_{\text {average }}$ & $k_{\min } / k_{\max }$ & $\kappa_{l}$ \\
\hline PtLaSb & 1.81 & 3.60 & 3.27 & 2.91 & 0.50 & 41.83 & 117.98 & 85.33 & 81.71 & 0.35 & 0.84 \\
RhLaTe & 2.32 & 3.47 & 3.52 & 3.12 & 0.66 & 36.22 & 109.79 & 103.74 & 83.25 & 0.33 & 1.21 \\
BiBaK & 1.62 & 1.96 & 3.02 & 2.21 & 0.54 & 36.06 & 34.54 & 23.41 & 31.34 & 0.65 & 2.26 \\
PCdNa & 6.53 & 2.31 & 5.59 & 4.85 & 0.35 & 86.60 & 39.41 & 47.04 & 57.68 & 0.46 & 2.27 \\
\hline \hline
\end{tabular}

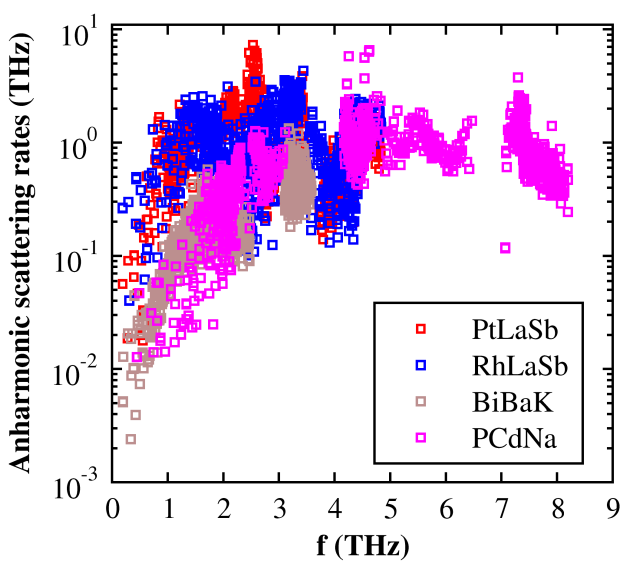

FIG. 7. Calculated $300 \mathrm{~K}$ anharmonic scattering rates for the four low thermal conductivity compounds. phonons are highest for the lowest thermal conductivity materials, specifically PtLaSb and RhLaSb, as may be expected. Finally, the ratios between the smallest to largest effective spring constant for PtLaSb, RhLaTe, BiBaK, and PCdNa are low, 0.35, 0.33, 0.65, and 0.46, respectively.

\section{SUMMARY AND CONCLUSIONS}

We investigated the thermal conductivity of halfHeusler semiconductors in relation to the phonon dispersions using different measures related in particular to rattling. We find that the thermal conductivity is correlated with average phonon frequency as expected and also surprisingly well with average effective spring constant. This is connected with the idea that weak bonding leads to greater anharmonic scattering. We constructed two measures based on local dynamics using the site aver- 
age phonon frequency from the projected phonon density of states. The first is a ratio of the lowest site average frequency to the highest. The second is a ratio of the lowest effective spring constant to the highest. Both of these correlate with thermal conductivity and are different from each other. For identifying the lowest thermal conductivity the first $\left(\omega_{\min } / \omega_{\max }\right)$ is somewhat better in this set of compounds. This measure corresponds to the idea that low rattling frequency is best. The other measure $\left(k_{\min } / k_{\max }\right)$, which measures bonding is also well correlated with thermal conductivity. We note that neither of these ratios scales with phonon velocity. We hope that these results are useful in providing understanding of rattling in relation to thermal conductivity and perhaps in screening materials for potential low thermal conductivity.

\section{ACKNOWLEDGMENTS}

Work at the University of Missouri was supported by the U. S. Department of Energy, Office of Science, Basic Energy Sciences, Award No. de-sc0019114. Work at ISSP and USTC was supported by National Science Foundation of China, Award 11774347. Z.F. gratefully acknowledges support from the China Scholarship Council (CSC). yshzhang@theory.issp.ac.cn

$\dagger$ singhdj@missouri.edu

1 C. Wood, Rep. Prog. Phys. 51, 459 (1988).

2 J. He and T. M. Tritt, Science 357, eaak9997 (2017).

3 T. M. Tritt, Science 283, 804 (1999).

${ }^{4}$ M. Beekman, D. T. Morelli, and G. S. Nolas, Nature Materials 14, 1182 (2015).

5 J. L. Cohn, G. S. Nolas, V. Fessatidis, T. H. Metcalf, and G. A. Slack, Phys. Rev. Lett. 82, 779 (1999).

6 T. Takabatake, K. Suekuni, T. Nakayama, and E. Kaneshita, Rev. Mod. Phys. 86, 669 (2014).

7 B. C. Sales, D. Mandrus, and R. K. Williams, Science 272, 5266 (1996).

8 G. Nolas, G. Slack, D. Morelli, T. Tritt, and A. Ehrlich, J. Appl. Phys. 79, 4002 (1996).

9 X. Shi, J. Yang, J. R. Salvador, M. Chi, J. Y. Cho, H. Wang, S. Bai, J. Yang, W. Zhang, and L. Chen, J. Am. Chem. Soc. 133, 7837 (2011).

10 G. S. Nolas, J. L. Cohn, and G. A. Slack, Phys. Rev. B 58, 164 (1998).

11 H. Luo, J. W. Krizan, L. Muechler, N. Haldolaarachchige, T. Klimczuk, W. Xie, M. K. Fuccillo, C. Felser, and R. J. Cava, Nature Comm. 6, 6489 (2015).

12 D. J. Singh and I. I. Mazin, Phys. Rev. B 56, R1650 (1997).

13 X. Shi, J. Yang, L. Wu, J. R. Salvador, C. Zhang, W. L. Willaire, D. Haddad, J. Yang, Y. Zhu, and Q. Li, Sci. Rep. 5, 14641 (2015).

14 J. Yang, L. Xi, W. Qiu, L. Wu, X. Shi, L. Chen, J. Yang, W. Zhang, C. Uher, and D. J. Singh, NPJ Comput. Mater. 2, 15015 (2016).

15 L. Lindsay, C. Hua, X. L. Ruan, and S. Lee, Materials Today Physics 7, 106 (2018).

16 B. C. Sales, B. C. Chakoumakos, D. Mandrus, and J. W. Sharp, J. Solid State Chem. 146, 528 (1999).

17 V. Keppens, D. Mandrus, B. C. Sales, B. C. Chakoumakos, P. Dai, R. Coldea, M. B. Maple, D. A. Gajewski, E. J. Freeman, and S. Bennington, Nature 395, 6705 (1998).

18 J. L. Feldman, D. J. Singh, I. I. Mazin, D. Mandrus, and B. C. Sales, Phys. Rev. B 61, R9209 (2000).

19 W. Li, L. Lindsay, D. A. Broido, D. A. Stewart, and N. Mingo, Phys. Rev. B 86, 174307 (2012).

${ }^{20}$ W. Li, J. Carrete, N. A. Katcho, and N. Mingo, Comput. Phys. Commun. 185, 1747 (2014).
21 A. Togo, L. Chaput, and I. Tanaka, Phys. Rev. B 91, 094306 (2015).

22 O. Hellman and D. A. Broido, Phys. Rev. B 90, 134309 (2014).

23 C. Uher, J. Yang, S. Hu, D. T. Morelli, and G. P. Meisner, Phys. Rev. B 59, 8615 (1999).

${ }^{24}$ G. Joshi, X. Yan, H. Wang, W. Liu, G. Chen, and Z. Ren, Adv. Energy Mater. 1, 643 (2011).

25 M.-S. Lee, F. P. Poudeu, and S. D. Mahanti, Phys. Rev. B 83, 085204 (2011).

26 M. Gurth, G. Rogl, V. V. Romaka, A. Grytsiv, E. Bauer, and P. Rogl, Acta Materialia 104, 210 (2016).

27 J. Zhou, H. Zhu, T. H. Liu, Q. Song, R. He, J. Mao, Z. Liu, W. Ren, B. Liao, D. J. Singh, Z. Ren, and G. Chen, Nature Comm. 9, 1721 (2018).

28 G. Samsonidze and B. Kozinsky, Adv. Energy Mater. 8, 1800246 (2018).

${ }^{29}$ H. Zhu, J. Mao, Y. Li, J. Sun, Y. Wang, Q. Zhu, Q. Song, J. Zhou, Y. Fu, R. He, T. Tong, Z. Liu, W. Ren, Z. Wang, J. Luo, A. Sotnikov, J. Bao, K. Nielsch, G. Chen, D. J. Singh, and Z. Ren, Nature Comm. 10, 270 (2019).

30 H. Zhu, J. Mao, Z. Feng, J. Sun, Q. Zhu, Z. Liu, D. J. Singh, Y. Wang, and Z. Ren, Science Adv. 5, eaav5813 (2019).

31 J. Carrete, W. Li, N. Mingo, S. Wang, and S. Curtarolo, Phys. Rev. X 4, 011019 (2014).

32 J. Shiomi, K. Esfarjani, and G. Chen, Phys. Rev. B 84, 104302 (2011).

33 S. R. Culp, S. J. Poon, N. Hickman, T. M. Tritt, and J. Blumm, Appl. Phys. Lett. 88, 042106 (2006).

34 S. Chen, K. C. Lukas, W. Liu, C. P. Opeil, G. Chen, and Z. Ren, Adv. Energy Mater. 3, 1210 (2013).

35 G. Ding, G. Y. Guo, and K. L. Yao, J. Phys. D: Appl. Phys. 48, 235302 (2015).

${ }^{36}$ P. Holuj, C. Euler, B. Balke, U. Kolb, G. Fiedler, M. M. Muller, T. Jaeger, E. Chavez Angel, P. Kratzer, and G. Jakob, Phys. Rev. B 92, 125436 (2015).

37 S. N. H. Eliassen, A. Katre, G. K. H. Madsen, C. Persson, O. M. Lovvik, and K. Berland, Phys. Rev. B 95, 045202 (2017).

38 B. Gong, Y. Li, F. Liu, J. Zhu, X. Wang, W. Ao, C. Zhang, J. Li, H. Xie, and T. Zhu, ACS Appl. Mater. Interfaces 11, 13397 (2019). 
${ }^{39}$ K. Berland, N. Shulumba, O. Hellman, C. Persson, and O. M. Lovvik, J. Appl. Phys. 126, 145102 (2019).

40 A. Putatunda and D. J. Singh, Materials Today Physics 8, 49 (2019)

41 S. Lee, K. Esfarjani, T. Luo, J. Zhou, Z. Tian, and G. Chen, Nature Comm. 5, 3525 (2014).

42 P. Hermet, R. M. A. amd E Theron, P. G. Yot, F. Salles, M. Tillard, and P. Jund, J. Phys. Chem. C 118, 22405 (2014).

43 T. Graf, C. Felser, and S. S. P. Parkin, Prog. Solid State Chem. 39, 1 (2011).

44 D. Bende, F. R. Wagner, and Y. Grin, Inorg. Chem. 54, 3970 (2015).

45 D. Bende, Y. Grin, and F. R. Wagner, Chemistry 20, 9702 (2014)

${ }^{40}$ Z. Feng, Y. Fu, A. Putatunda, Y. Zhang, and D. J. Singh, Phys. Rev. B 100, 085202 (2019).

47 F. Legrain, J. Carrete, A. van Roekeghem, G. K. H. Madsen, and N. Mingo, J. Phys. Chem. B 122, 625 (2017).
48 P. E. Blöchl, Phys. Rev. B 50, 17953 (1994).

49 G. Kresse and J. Furthmuller, Comput. Mater. Sci. 6, 15 (1996).

50 M. Omini and A. Sparavigna, Phys. Rev. B 53, 9064 (1996).

51 A. Togo, F. Oba, and I. Tanaka, Phys. Rev. B 78, 134106 (2008).

52 O. Hellman, P. Steneteg, I. A. Abrikosov, and S. I. Simak, Phys. Rev. B 87, 104111 (2013).

53 J. Callaway, Phys. Rev. 113, 1046 (1959).

54 G. S. Nolas, T. J. R. Weakley, J. L. Cohn, and R. Sharma, Phys. Rev. B 61, 3845 (2000).

55 L. Lindsay, D. A. Broido, and T. L. Reinecke, Phys. Rev. Lett. 111, 025901 (2013).

56 S. Mukhopadhyay, D. Bansal, O. Delaire, D. Perrodin, E. Bourret-Courchesne, D. J. Singh, and L. Lindsay, Phys. Rev. B 96, 100301(R) (2017). 